\title{
Clinical risk management in radiotherapy: Published guidelines and own experience
}

\author{
Peter Winkler", Tanja Langsenlehner, Edith Geyer, Astrid Oechs, Andreas Uller, Karin Kapp \\ From Safety in hospitals: from strategy to implementation Annual Scientific Meeting 2015 \\ Graz, Austria. 29-30 September 2015
}

\section{Background}

Radiotherapy (RT) is one of the major treatment options in cancer management. The process of RT is complex and incorporates the use of highly specialized technical equipment. Therefore quality assurance (QA) procedures have always been an inclusive part of RT. Due to the rapidly increasing complexity during the last years, comprehensive QA has become even more of an issue, including treatment planning software, information handling and treatment delivery. Obviously methods from clinical risk management (RM) can be well adapted and adopted for use in radiotherapy. In this paper we aim to discuss currently available guidelines on RM in radiotherapy and to present risk assessment and process evaluation activities involved in the RM-implementation process at our department.

\section{Material and methods}

We searched databases fur publications on incidents and risk management in radiotherapy. Statistics from RTspecific incident reporting systems were analysed.

A RM system at our clinic was established according to the national guidelines. By means of a scenario analysis the substantial risks were assessed. If necessary, adjustments of the existing processes were initiated. All treatment-related processes, where incidents could lead to hazards or harm, were reviewed using Failure Mode and Effects Analysis and their level of risk was analysed. Activities were set to support an open and permissive risk culture in the department: quick and traceable handling of suggestions for improvement, naming of persons of trust and comprehensive information for all clinic staff. The internal communication plan was adjusted and supplemented by RM-specific elements.

\section{Results}

Between 1976 and 2007, 3125 patients were reported to be affected by adverse events following radiotherapy incidents. 38 patients died due to toxicity following radiation overdose [1]. Approximately 4500 near misses were reported in publically available databases for the period 1992 to 2007. There are no current figures available, that could illustrate the influence of the widely spread usage of technically high complex treatments since that time. The majority of incidents were related to data handling issues or multiple influences (73\%), followed by treatment delivery (18\%) or treatment planning (9\%). The analysis of our in-house incident reports yielded a similar distribution.

\section{Conclusion}

Recommendations on RM in radiotherapy have been published by the WHO [1] and the European Commission [2]. A complete RM-system should encompass all involved steps of the process, including initial choice of treatment and dose prescription.

\section{Competing interests}

None

Published: 30 October 2015

\section{References}

1. World Health Organization: Radiotherapy risk profile. Technical Manual Geneva; 2008.

2. European Commission: Radiation Protection $N^{\circ} 181$. General guidelines on risk management in external beam radiotherapy Luxembourg; 2015.

doi:10.1186/2056-5917-1-S1-A28

Cite this article as: Winkler et al:: Clinical risk management in

radiotherapy: Published guidelines and own experience. Safety in Health 2015 1(Suppl 1):A28.

\footnotetext{
* Correspondence: peter.winkler@medunigraz.at

Department of Therapeutic Radiology and Oncology, Medical University of Graz, Graz Austria
} 